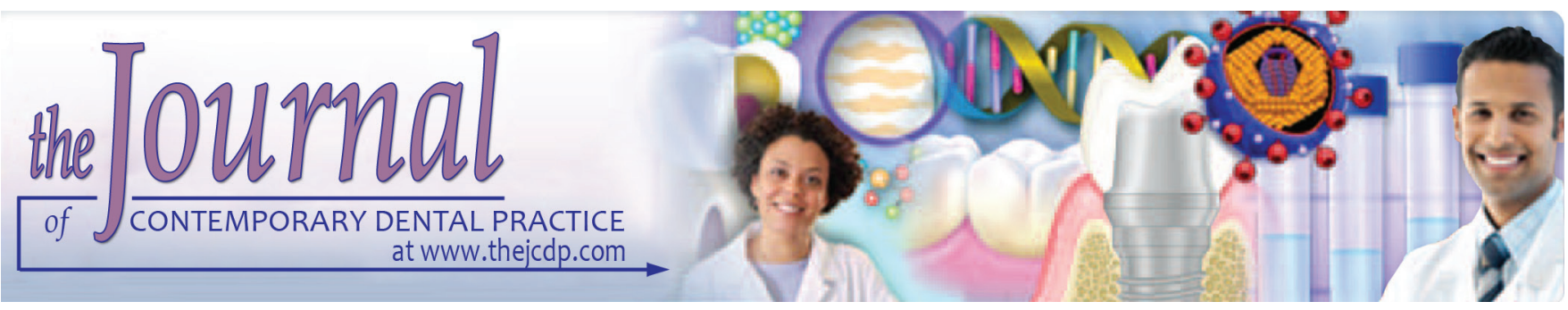

\title{
Antimicrobial Effect of an Experimental Glass lonomer Cement against Pathogens associated with Deep Carious Lesions
}

${ }^{1}$ Kalepu Vamsi, ${ }^{2}$ Fawaz Siddiqui

\begin{abstract}
Aim: To study the antimicrobial effect of chlorhexidine diacetate (CHX-D)-modified type II glass ionomer cement (GIC) against the two predominant deep caries microorganisms, namely Lactobacillus casei and Actinomyces viscosus.
\end{abstract}

Materials and methods: An experimental GIC (ex-GIC) was prepared by mixing $\mathrm{CHX}-\mathrm{D}$ powder with the powder of type II GIC to obtain $1 \%(w / w)$ concentration of CHX-D in the GIC. Antibacterial activity of this ex-GIC was tested against $L$. casei and $A$. viscosus using the agar diffusion method. The ex-GIC specimens were tested in their unset and set forms for each bacterium. For the unset group, specimens were placed in each agar plate immediately after manipulation and for the set group, specimens were placed in each agar plate, 1 hour after manipulation. The inhibition zones on the agar plate were recorded in millimeters immediately on placement of the specimen in the agar plate and after 48 hours. The reading was recorded and statistically analyzed for significant difference.

Results: Mann-Whitney U test showed statistically significant difference in the inhibition zones produced by ex-GIC against $L$. casei and $A$. viscosus when both were compared in unset $(p$-value $=0.002)$ and set $(p$-value $=0.031)$ groups. For both the groups, the zone of inhibition against $L$. casei was greater. Though the unset group recorded wider zone of inhibition, the difference was not significant when compared with the respective set group. This was true for both the bacterial groups.

Conclusion: The 1\% CHX-D-modified type II GIC showed antibacterial property against $L$. casei and $A$. viscosus and significantly higher activity against $L$. casei.

Clinical significance: Addition of $1 \% \mathrm{CHX}-\mathrm{D}$ to type II GIC showed evidence of antibacterial activity against organisms

1,2Department of Pediatric Dentistry, Penang International Dental College, Penang, Malaysia

Corresponding Author: Fawaz Siddiqui, Department of Pediatric Dentistry, Penang International Dental College, Penang Malaysia, Phone: +60199442928, e-mail: drfawazsiddiqui@ gmail.com found in deep carious lesion and therefore may exhibit superior antimicrobial efficiency when used as an intermediate therapeutic restoration in deep cavities.

Keywords: Actinomyces viscosus, Antibacterial, Chlorhexidine, Glass ionomer cement, Lactobacillus casei.

How to cite this article: Vamsi K, Siddiqui F. Antimicrobial Effect of an Experimental Glass lonomer Cement against Pathogens associated with Deep Carious Lesions. J Contemp Dent Pract 2018;19(7):824-829.

Source of support: Nil

Conflict of interest: None

\section{INTRODUCTION}

Dental caries is not an infectious disease, which needs to be cured by removing bacteria. ${ }^{1}$ It is merely a reflection of an ecological imbalance between the commensal microorganisms and the acidogenic/aciduric microorganisms found within the dental biofilm as described originally in the ecological plaque hypothesis. ${ }^{2}$ In such a biofilm, the acidogenic and aciduric bacterial population prevails, causing net mineral loss from the hard tissues of the tooth by demineralization. Traditional approach for management of carious lesion was therefore focused on the removal of demineralized bacterial-infected tooth tissue to ensure control of caries progression and in effect protect the pulp from getting infected. Unfortunately, such mechanical removal of cariously infected tooth tissue was found to be ineffective in eliminating all the bacteria from the cavity and failed to heal and control the lesion activity. ${ }^{3}$ Also, excavation of caries may not always eliminate all microorganisms in the residual tissues. ${ }^{4}$ Moreover, complete caries excavation, now referred to as nonselective caries removal to hard dentin, may cause stress to the pulp during deep caries excavation, leading to failure of intact restoration due to pulpal inflammation. 
To overcome this issue, selective caries removal to soft dentin and stepwise caries removal were advocated. These techniques maintain the pulp vitality by minimizing carious pulpal exposure during deep caries excavation and thus avoiding endodontic treatment. This is an important clinical technique in primary dentition where the likelihood of pulpal exposure during deep caries excavation is high because of large pulp organ protected by thin enamel and dentin. After partial caries removal as in stepwise caries excavation, many microorganisms can still remain alive in the dentin substrate, even in the presence of a standard sealing. ${ }^{5-8}$

The microorganisms recovered from deep dentinal carious lesions are diverse and different from those recovered from carious lesion in enamel. The microbial flora in dentinal carious lesion are facultative and obligate-anaerobic bacteria belonging to the genera Actinomyces, Bifidobacterium, Eubacterium, Lactobacillus, Parvimonas, and Rothia. Streptococci which are involved in initiating carious lesion in enamel are recovered less frequently from dentinal lesions. The cariogenic bacteria may harbor in the dentinal tubules and are protected by the smear layer. Such continual bacterial presence, in conjunction with the lack of an airtight seal between the restoration and the cavity walls, may cause the development of recurring caries. ${ }^{9}$ Thus, incomplete removal of caries during tooth preparation or microleakage between tooth-restoration interface could result in a restoration with underlying viable microorganisms. ${ }^{10}$

In pediatric dentistry, there is a need for a restorative material which has antibacterial properties and also has adhesive bonding to the tooth structure. A probable solution to this clinical problem could be the use of dental materials which have a good bacteriostatic property. ${ }^{11}$ Clinical use of restorative materials with an inhibiting action on microbial growth can have two benefits: (a) Antibacterial substances can extend the longevity of restorations and (b) they can help alleviate postoperatory discomfort. ${ }^{12}$ No other dental material except GIC fulfills this criterion, but the antibacterial effect of glass ionomer is limited and confined to release of fluoride. There is a need to potentiate the antibacterial property of GIC, so that it can be used as a restorative material of choice in nonselective caries removal to soft dentin in primary teeth.

Combining GIC with an antiseptic agent could result in a restorative material with superior antibacterial property. Chlorhexidine diacetate, when incorporated into GIC, was found to increase the antibacterial effect of GIC in vitro. ${ }^{13-17}$ Many studies have investigated the antibacterial activity of CHX-modified GIC against Streptococcus mutans, an agent responsible for initiating caries. There is very limited research on the antibacterial effect of CHX-D-modified GIC on bacteria, which are most commonly isolated from deep and advancing caries lesion. Therefore, the aim of this study was to assess the antimicrobial activity of CHX-D modified type II GIC (ex-GIC) against pathogens associated with progressing deep caries lesion, namely Lactobacilli casei and A. viscosus. The outcome of this study is anticipated to provide knowledge for the application of concept of minimal invasive dentistry in primary teeth.

\section{MATERIALS AND METHODS}

The design of this study was an experimental lab-based study under strict environmental control. The ex-GIC was prepared by incorporating $10 \mathrm{mg}$ of CHX-D powder (Smaart Pharmaceuticals Company, Jalgoan, India) to 1,000 mg of type II GIC powder (GC Corporation, Tokyo, Japan), to obtain $1 \% \mathrm{w} / \mathrm{w}$ concentration of CHX-D in the GIC formulation. An electronic balance machine was used to precisely weigh both the powders. The powder was manually mixed ensuring uniform distribution of the CHX-D powder in the GIC powder. The GIC liquid was not changed or modified.

The antibacterial activity of the ex-GIC was assessed by agar diffusion test against the L. casei [American Type Culture Collection (ATCC) 393] and A. viscosus (ATCC 15987) obtained from Hi Media Laboratories Pvt. Ltd., Mumbai, India. All procedures were performed in a laminar cabinet under aseptic conditions. The ex-GIC specimens were divided into four groups namely, group Ia-L. casei (unset); group $\mathrm{Ib}-L$. casei (set); group IIa-A. viscosus (unset); and group IIb-A. viscosus (set). For the unset group, eight specimens were placed in each agar plate immediately after manipulation and before they set. For the set group, eight specimens were placed in each agar plate, 1 hour after they were manipulated. The inhibition zones on the agar plate were recorded in millimeters immediately on placement of the specimen in the agar plate and after 48 hours. The readings were recorded and statistically analyzed for significant difference.

\section{Agar Diffusion Test}

The bacterial strains that we obtained from stock cultures were stored in $50 \%$ glycerol at $-20^{\circ} \mathrm{C}$. The cultivation was then done in brain heart infusion (BHI) broth at $37^{\circ} \mathrm{C}$ and a loopful of inoculum was transferred to $10 \mathrm{~mL}$ of $\mathrm{BHI}$ broth and incubated for 48 hours. The BHI agar plate was inoculated with $350 \mathrm{~mL}$ bacterial suspension that was spread out evenly and left for 30 minutes at room temperature.

Wells of $6 \mathrm{~mm}$ diameter and $2 \mathrm{~mm}$ depth were punched into the agar plates; eight such wells were prepared in each agar plate. Two agar plates were prepared 
for each group of bacteria. Powder and liquid of ex-GIC were proportioned and mixed according to manufacturer's instructions given for type II GIC with a sterile agate spatula (one drop of liquid for one scoop).

For the unset group, the cement was inserted immediately with a sterile dental instrument into the wells of the specific bacterium-inoculated BHI agar plate. In total, 16 ex-GIC specimens were placed for each strain of bacteria.

For the set specimen, the cement was manipulated and placed into holes prepared in sterile brass mold. These holes were of the same dimensions $(6 \mathrm{~mm} \times 2 \mathrm{~mm})$ as that of the well in the agar plate. The specimens were then covered with sterile glass slides and allowed to set for 60 minutes at room temperature. A total of 32 specimens were prepared and they were randomly divided equally into groups $\mathrm{Ib}$ and $\mathrm{IIb}$. The set specimens were then placed onto the BHI agar plate inoculated with specific bacterial strain.

The halo around the specimen indicating the zone of inhibition was measured in millimeters with a digital caliper, immediately after the placement of the specimens in the agar plates, by a single observer. Plates were then incubated at $37^{\circ} \mathrm{C}$ for 48 hours. After 48 hours, the zones of inhibition produced around each specimen in all the groups were recorded in millimeters using the same digital caliper and by the same observer. The readings were recorded at three random places. The mean of the three readings was used as the final measurement.

The results were tabulated and statistically analyzed using Mann-Whitney U test for testing the hypothesis that there is no difference between the widths of zone of inhibition in the independent samples.

\section{RESULTS}

The mean zone of inhibition recorded from the different groups is shown in Table 1. There was no zone of

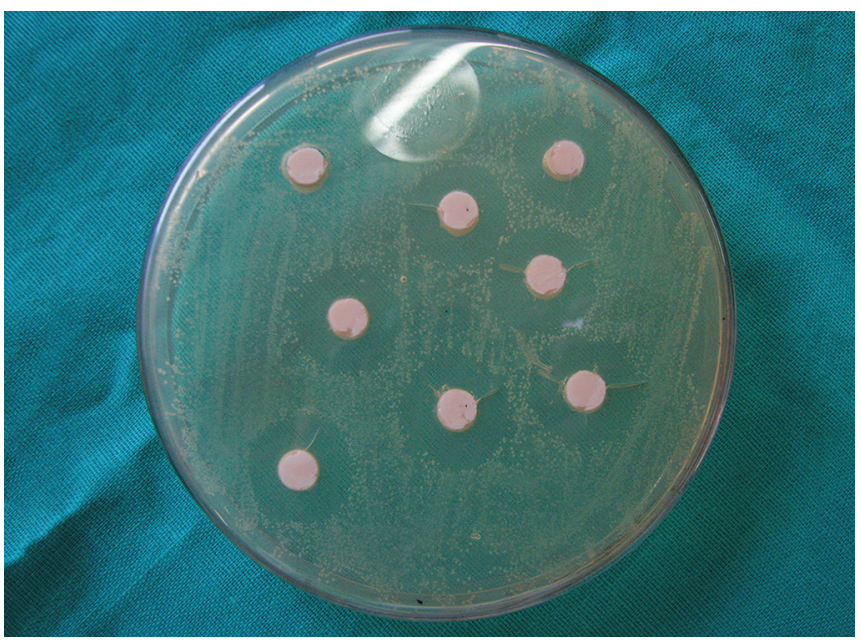

Fig. 1: Inhibition zones for group la
Table 1: The mean value of zone of inhibition recorded for the unset and set ex-GIC against the two organisms

\begin{tabular}{lll}
\hline Organism & Unset ex-GIC & Set ex-GIC \\
\hline Lactobacillus casei & $17.62(01.76)^{1}$ & $17.00(1.16)^{1}$ \\
Actinomyces viscosus & $14.12(0.83)^{2}$ & $14.00(0.75)^{2}$ \\
\hline
\end{tabular}

Values in parentheses denote the standard deviation; Same superscript number denotes nonsignificant difference between groups; Different superscript number denotes significant difference between groups

inhibition recorded $(0 \mathrm{~mm})$, immediately after the placement of the specimens in the agar plates in all the groups. After 48 hours, it was found that the zone of inhibition in the unset ex-GIC was widest against L. casei $(17.62 \pm 1.76$ $\mathrm{mm})$, followed by group $\mathrm{Ib}(17.00 \pm 1.16 \mathrm{~mm})$, group IIa $(14.12 \pm 0.83 \mathrm{~mm})$ and narrowest for group $\mathrm{IIb}(14.00$ $\pm 0.75 \mathrm{~mm})$.

When groups Ia and $\mathrm{Ib}$ were compared, it was found that there was no statistically significant difference between the unset and the set specimen against $L$. casei $(p=0.69)$. A similar result was obtained when unset and set ex-GIC groups were compared for zone of inhibition on $A$. viscosus ( $\mathrm{p}=0.78$ ). This suggested that though there was an increase in the antibacterial activity immediately after mix, the setting reaction did not greatly alter the antibacterial effect of ex-GIC.

On comparing the effect of unset specimen between the bacterial strains, it was found that unset specimen inhibited L. casei significantly more than A. viscous ( $p=0.002)$. The same inference was drawn when set specimens were compared between the bacterial strains $(\mathrm{p}=0.03)$. Lactobacillus casei showed larger zones of inhibition (Figs 1 and 2).

\section{DISCUSSION}

Restoration of deeply carious primary tooth poses a clinical challenge because deep caries excavation in a primary

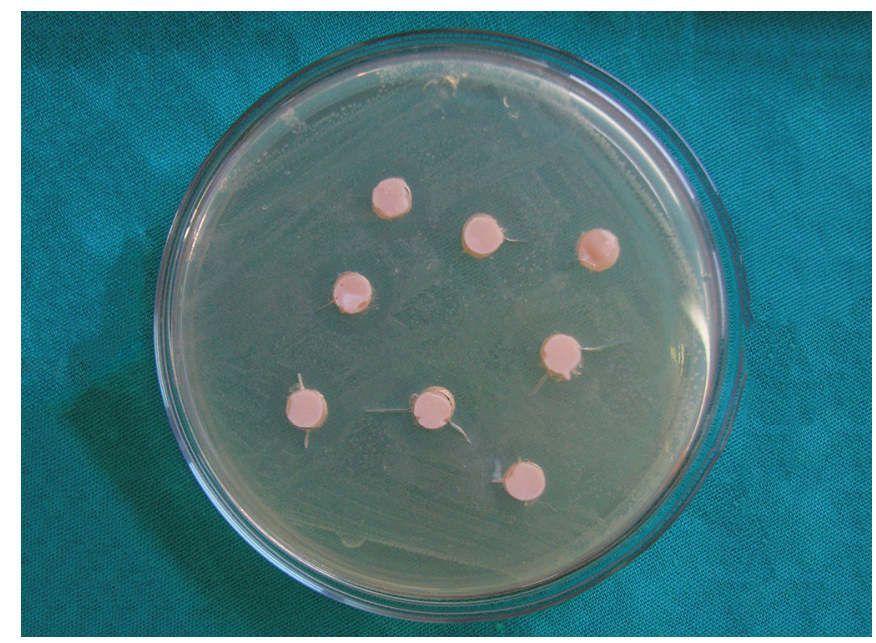

Fig. 2: Inhibition zones for group Ila 
tooth has an inherent risk of pulpal exposure and thereby converting an anticipated restorative procedure to a more complex pulpotomy/pulpectomy procedure, thereby increasing the cost of the treatment and number of dental visits. The International Caries Consensus Collaboration recommends that pulp vitality should be maintained by avoiding dentin excavation close to the pulp, so minimizing the risk of pulpal exposure. ${ }^{18}$ This is done by selective caries removal to soft dentin. The only prerequisite here is that this soft dentin must be peripherally sealed from the oral environment by adequate thickness of an adhesive restoration. Further, to improve the long-term success of the tooth-restoration complex, enough soft dentin must be removed to place a durable restoration of sufficient thickness and resiliency, while maintaining sufficient tooth support around the restoration. This may be difficult to achieve each time when managing deep carious lesion in primary teeth because pulp exposure inadvertently happens because of high pulp horns and less enamel and dentin thickness as compared with permanent teeth. Microleakage in primary dentition is an important consideration because the seepage of irritants around the restoration and through the thin dentin may produce irreversible pulp damage. ${ }^{19}$ In underdeveloped and developing countries, the burden of curative treatment of caries is high and further iatrogenic endodontic treatment will jeopardize the efforts. There is a need for a restorative material which will not only provide adhesive seal but will also have antibacterial activity against pathogens involved in deep caries progression. This could greatly increase the prognosis during selective caries removal in soft dentin.

In the etiology of early childhood caries, many strains of microorganism have been isolated. Streptococcus mutans have been found to play a major role because it provides the thick tenacious matrix of biofilms in which it multiplies and produces acid. This acid production not only demineralizes the hard tissues of the tooth but also provides a protective niche and acid milieus within which other acidogenic and aciduric organisms thrive and colonize. Streptococcus mutans may well be a biofilm initiator (and also a potent acid producer) that paves the way for other cariogenic bacteria to become dominant, possibly at the expense of $S$. mutans itself, as the biofilm matures. ${ }^{20}$ Among the other cariogenic bacteria, Lactobacilli and Actinomyces strains have been frequently isolated from deep caries lesions and they are believed to be the pioneering microorganisms in the caries progress, especially in dentin. Hence, this study included L. casei and $A$. viscosus as the test organism to assess the antibacterial efficacy of ex-GIC.

The modern-day GIC is the only dental restorative material which can bond chemically to the enamel and dentin. It acts as a biomimetic material and a reservoir for fluoride release. This makes it ideal for peripheral seal of deep cavitated lesions. Earlier studies suggested that GIC is antimicrobial because of their fluoride release and/or their acidity. ${ }^{21-25}$ It has been well established that fluoride is released from $\mathrm{GIC}^{26-28}$ and the material has a low $\mathrm{pH}$ while setting, but the results of previous investigations related to the antibacterial effects of both fluoride and low $\mathrm{pH}$ are still controversial. ${ }^{29}$ Also, the reduction in bacterial counts obtained by placing conventional GICs in cavities is not reliable; therefore, innovation was required to potentiate the antibacterial action of GIC.

Based on the previous study which had confirmed bacterial growth inhibition by conventional GIC, ${ }^{30}$ the present study modified the type II restorative GIC, by incorporation of $1 \%$ chlorhexidine acetate powder to the GIC powder. Chlorhexidine was chosen over other antibiotic powders because chlorhexidine is a broad spectrum antimicrobial which acts as bactericidal as well bacteriostatic agent against both Gram-negative and Gram-positive bacteria. Its property of susbstantivity increases its binding to tooth structure and increases the contact time with the tooth structure. Sugar transport and acid production in oral bacteria are also affected by CHX. The present study used the diacetate form of CHX because incorporation of CHX-D into GICs was found to increase the antimicrobial effect of GIC without seriously compromising the physical properties of the original material. ${ }^{13,14}$ The diacetate form is a more stable material, not prone to decomposition, and can be easily blended with glass ionomer powder. The presence of CHX-D in the GIC does not contribute to the formation of the glass ionomer network concentration and therefore can weaken the scaffold and compromise the physical properties of the antibacterial glass ionomer if used in higher concentration in an attempt to increase the antibacterial property of the ex-GIC. ${ }^{31}$ Takahashi et al ${ }^{15}$ showed with a high-performance liquid chromatography test that there was very little CHX-D released from their ex-GIC formulations and concluded that a $1 \% \mathrm{CHX}-\mathrm{D}$ addition was optimal to give appropriate physical and antibacterial properties. Therefore, the concentration of CHX-D in this study was kept at $1 \%$. Type II GIC was preferred over type IX GIC, because of personal preferences. The authors find that well-manipulated type II GIC provides similar restorative longevity in primary teeth as compared with type IX GIC in single surface occlusal restorations. Further, there are no recommendations for restoring teeth with particular restorative material after using selective caries removal in soft dentin. ${ }^{1}$

In our study, we used agar plate diffusion method, as it allowed both set and unset materials to be assayed rapidly and easily with a large number of specimens. However, 
this test has to be interpreted with caution because it does not simulate the clinical condition of carious lesion where multiple species of bacteria grow in a complex biofilm.

The present study investigated the antibacterial activity of unset ex-GIC on bacteria found in deep carious lesions. This was thought to mimic the clinical situation and hence be of more clinical significance. Though statistically there was no significant difference in the mean zone of inhibition between unset and set restoration, this study did demonstrate that unset ex-GIC had wider zone of inhibition in both the test bacteria. The addition of CHX-D diacetate to type II GIC had antibacterial properties against both $A$. viscosus and $L$ casei, and significantly greater activity against $L$. casei. The wider zone of inhibition of unset specimen can be explained by the low $\mathrm{pH}$ of unset GIC. As the chelation reaction progresses, the leaching of chlorhexidine may also have reduced.

The results of the present study are in accordance with a similar study conducted by Türkün et $\mathrm{al}^{31}{ }^{31}$ who found that for L. acidophilus, the greatest antibacterial effect was observed with diacetate group. The $2.5 \%$ group of CHX-D showed antibacterial activity up to 90 days against $S$. mutans and up to 60 days against L. acidophilus. There is a very limited research on antibacterial activity of 1\% CHX-D-modified type II GIC on Actinomyces and Lactobacillus.

The results of this study may not be directly relevant to clinical practice because it does not discuss the compressive strength of the set material and rate of release of fluoride from the ex-GIC. The inhibitory zones were measured only after 48 hours and therefore it is uncertain whether antibacterial action gradually increased or decreased over the 2 days and how it would have fared over the next few days. The present study does provide the stepping stone by proving that $1 \%$ CHX-D-modified GIC has antibacterial effect on commonly isolated deep caries-associated pathogens. The study also assesses the effect of unset ex-GIC. Further research is recommended to investigate this ex-GIC, with an objective to develop it as a cost-effective restorative material which will provide predictable outcome in restoration of primary teeth using selective caries removal in soft dentin.

\section{CONCLUSION}

Chlorhexidine-modified GIC's antibacterial effect has shown promising results against $S$. mutans, which is responsible for initiating superficial caries, but not against L. casei and A. viscosus, which are the predominant organisms of deep carious lesions. This in vitro study confirmed the antibacterial effect of $1 \%$ CHX-D modified GIC against both these organisms, thus extending its use in deep caries excavation, where additional antibacterial effect may be required. Within the limitations of this study, we found that addition of as less as 1\% CHX-D diacetate to conventional GIC was effective in inhibiting $L$. casei and A. viscosus, suggesting further investigation on its use in deep caries management, in minimal invasive dentistry for children.

\section{CLINICAL SIGNIFICANCE}

Addition of 1\% CHX-D to type II GIC showed evidence of antibacterial activity against organisms found in deep carious lesion and therefore may find use as an intermediate restoration in deep cavities to sterilize the cavities and protect the pulp and in single-visit stepwise caries excavation in primary teeth.

\section{REFERENCES}

1. Banerjee A, Frencken JE, Schwendicke F, Innes NPT. Contemporary operative caries management: consensus recommendations on minimal invasive caries removal. Br Dent J 2017 Aug;223(3):215-222.

2. Marsh, PD.; Martin, MV. Oral microbiology. 5th ed. Edinburg (NY): Elsevier; 2009. p. 222.

3. Lennon AM, Attin T, Martens S, Buchalla W. Fluorescenceaided caries excavation (FACE), caries detector and conventional caries excavation in primary teeth. Pediatr Dent 2009 Jul-Aug;31(4):316-319.

4. Kidd EA, Joyston-Bechal S, Beighton D. The use of a caries detector dye during cavity preparation: a microbiological assessment. Br Dent J 1993 Apr;174(7):245-248.

5. Bjorndal L, Larsen T. Changes in the cultivable flora in deep carious lesions following a stepwise excavation procedure. Caries Res 2000 Nov-Dec;34(6):502-508.

6. Weerheijm KL, Kreulen CM, de Soet JJ, Groen HJ, van Amerongen WE. Bacterial counts in carious dentine under restorations: 2-year in vivo effects. Caries Res 1999;33(2):130-134.

7. Pinto AS, de Araujo FB, Franzon R, Figueiredo MC, Henz S, Garcia-Godoy F, Maltz M. Clinical and microbiological effect of calcium hydroxide protection in indirect pulp capping in primary teeth. Am J Dent 2006 Dec;19(6):382-387.

8. Duque C, Negrini Tde C, Sacono NT, Spolidorio DM, de Souza Costa CA, Hebling J. Clinical and microbiological performance of resin-modified glass-ionomer liners after incomplete dentine caries removal. Clin Oral Invest 2009 Dec;13(4):465-471.

9. Bergenholtz G, Cox CF, Loesche WJ, Syed SA. Bacterial leakage around dental restorations: its effect on the dental pulp. J Oral Pathol 1982 Dec;11(6):439-450.

10. DeSchepper EJ, Thrasher MR, Thurmond BA. Antibacterial effects of light cured liners. Am J Dent 1989 Jun;2(3):74-76.

11. Herrera M, Castillo A, Baca A, Carrion P. Antibacterial activity of glass-ionomer restorative cements exposed to cavityproducingmicroorganisms. OperDent1999Sep-Oct;24(5)286-291.

12. Herrera M, Castillo A, Bravo M, Liebana J, Carrion P. Antibacterial activity of resin adhesives, glass ionomer and resin-modified glass ionomer cements and a compomer in contact with dentin caries samples. Oper Dent 2000 Jul-Aug;25(4):265-269.

13. Jedrychowski JR, Caputo AA, Kerper S. Antibacterial and mechanical properties of restorative materials combined with chlorhexidines. J Oral Rehabil 1983 Sep;10(5):373-381. 
14. Ribeiro J, Ericson D. In vitro antibacterial effect of chlorhexidine added to glass ionomer cements. Scand J Dent Res 1991 Dec;99(6):533-540.

15. Takahashi $Y$, Imazato S, Kaneshiro AV, Ebisu S, Frencken JE, Tay FR. Antibacterial effects and physical properties of glass-ionomer cements containing chlorhexidine for the ART approach. Dent Mater 2006 Jul;22(7):647-652.

16. Deepalakshmi M, Poorni S, Miglani R, Rajajmani I, Ramachandran S. Evaluation of the antibacterial and physical properties of glass ionomer cements containing chlorhexidine and cetrimide: an in-vitro study. Indian J Dent Res 2010 Oct-Dec;21(4):552-556.

17. Hoszek A, Ericson D. In vitro fluoride release and the antibacterial effect of glass ionomer containing chlorhexidine gluconate. Oper Dent 2008 Nov-Dec;33(6):696-701.

18. Schwendicke F. Contemporary concepts in caries tissue removal: a review. J Esthet Restor Dent 2017 Nov;29(6):403-408.

19. Siddiqui F, Karkare S. Sealing ability of nano-ionomer in primary teeth: an ex vivo study. Int J Clin Pediatr Dent 2016 Jul-Sep;9(3):209-213.

20. Hajishengallis E, Parsaei Y, Klein MI, Koo H. Advances in the microbial etiology and pathogenesis of early childhood caries. Mol Oral Microbiol 2017 Feb;32(1):24-34.

21. Ferreira GL, Alves LA, Jovito VC, de Carvalho FG, de Castro RD. Antibacterial activity of glass ionomer cements on cariogenic bacteria-an in vitro study. Int J Dent Clin 2011 Jan; 3(3):1-3.

22. Mahesk Kumar M, Mithun Pai BH, Prashant GM, Subba Reddy VV, Mohan Das U, Madura C, Chandu GN.
Antibacterial properties of fluoride releasing glass ionomer cements (GICs) and pit and fissure sealants on Streptococcus mutans. Int J Clin Pediatr Dent 2010 May-Aug;3(2):93-96.

23. Mc Comb D, Ericson D. Antimicrobial action of new, proprietary lining cements. J Dent Res1987 May;66(5):1025-1028.

24. DeSchepper EJ, White RR, von der Leh W. Antibacterial effect of glass ionomers. Am J Dent 1989 Apr;2(2):51-56.

25. Vermeersch G, Leloup G, Delmée M, Vreven J. Antibacterial activity of glass ionomer cements, compomers and resin composites: relationship between acidity and material setting phase. J Oral Rehabil 2005 May;32(5):368-374.

26. Weerheijm KL, de Soet JJ, van Amerongen WE, de Graaff J. The effect of glass ionomer cement on carious dentine: an in vivo study. Caries Res 1993 Feb;27(5):417-423.

27. Miller BH, Komatsu H, Nakajima H, Okabe T. Effect of glass ionomer manipulation on early fluoride release. Am J Dent 1995 Aug;8(4):182-186.

28. Tam LE, Chan GP, Yim D. In vitro caries inhibition effects by conventional and resin-modified glass-ionomer restorations. Oper Dent 1997 Jan-Feb;22(1):4-14.

29. Dunne SM, Goolnik JS, Millar BJ, Seddon RP. Caries inhibition by a resin modified and a conventional glass ionomer cement, in vitro. J Dent 1996 Jan-Mar;24(1-2):91-94.

30. Botelho MG. Compressive strength of glass ionomer cements with dental antibacterial agents. SADJ 2004 Mar;59(2):51-53.

31. Türkün LS, Türkün M, Ertuğrul F, Ateş M, Brugger S. Long-term antibacterial effects and physical properties of a chlorhexidine-containing glass ionomer cement. J Esthet Restor Dent 2008 Feb;20(1):29-45. 